\title{
Detect-and-Avoid Alerting Performance for High-Speed UAS and Non-Cooperative Aircraft
}

\author{
Andrew C. Cone ${ }^{1}$, and M. Gilbert $\mathrm{Wu}^{2}$ \\ NASA Ames Research Center, Moffett Field, CA 94035, USA \\ Seungman Lee ${ }^{3}$ \\ Crown Consulting Inc., NASA Ames Research Center, Moffett Field, CA 94035, USA
}

This paper investigates the alerting timeline and required surveillance volume for four candidate Detect and Avoid (DAA) Well Clear (DWC) definitions for Unmanned Aircraft Systems (UAS) and non-cooperative visual flight rules (VFR) traffic. The candidates are proposed in previous work that analyzed unmitigated collision risks and maneuver initiation ranges for UAS carrying low cost, size, weight, and power radar and that fly with true airspeeds under $100 \mathrm{kts}$. This analysis extends the maximum UAS true airspeed from $100 \mathrm{kts}$ to 291 kts. Overall, the results from this paper suggest that the smaller DWC definitions do not adversely affect average alerting times. Among the candidate DWC's examined, DWC2 has the potential of reducing the surveillance range requirement the most while maintaining the same alerting timeline. DWC2 is defined as a cylinder with a horizontal radius of 2,200 feet and a vertical range of 450 feet above and below the UAS.

\section{Nomenclature}

$\begin{array}{ll}\text { AGL } & =\text { above ground level } \\ \text { CPA } & =\text { closest point of approach } \\ \text { Low C-SWaP } & =\text { low cost, size, weight, and power } \\ \text { DAA } & =\text { detect and avoid } \\ \text { DWC } & =\text { detect-and-avoid well clear } \\ \text { FAA } & =\text { Federal Aviation Administration } \\ \text { DMOD } & =\text { distance modification } \\ \text { HMD } & =\text { projected horizontal miss distance } \\ \text { IFR } & =\text { instrument flight rules } \\ \text { LoDWC } & =\text { loss of detect and avoid well clear } \\ \text { MOPS } & =\text { minimum operational performance standards } \\ \text { MSL } & =\text { mean sea level } \\ \text { NMAC } & =\text { near mid-air collision } \\ \text { RADES } & =\text { RADAR Evaluation Squadron } \\ \text { SC-228 } & =\text { RTCA Special Committee 228 } \\ \text { TAS } & =\text { true airspeed } \\ \text { TSO } & =\text { technical standard order } \\ \text { UAS } & =\text { unmanned aircraft system } \\ \text { VFR } & =\text { visual flight rules } \\ h & =\text { altitude separation between two aircraft } \\ r & =\text { current horizontal range between two aircraft } \\ \dot{r} & =\text { rate of change of horizontal range between two aircraft } \\ \tau_{\text {mod }} & =\text { modified tau }\end{array}$

\footnotetext{
${ }^{1}$ Research Engineer, Aviation Systems, AIAA Member

${ }^{2}$ Research Engineer, Aviation Systems, AIAA Member

${ }^{3}$ Senior Research Scientist, Senior AIAA Member
} 


\section{Introduction}

Detect-and-avoid (DAA) systems serve as a critical module in enabling integration of Unmanned Aircraft System (UAS) operations in the National Airspace System (NAS). A DAA system provides surveillance, alerts, and maneuver guidance to keep a UAS "well clear" of other aircraft $[1,2]$. In the United States, simulation tests as well as flight tests have provided supporting information for defining a DAA Well Clear [1,3] (DWC) and requirements for the alerting and maneuver guidance performance [4-8], on which surveillance requirements were derived and based. The parameters used to define DWC are explained in more detail in section II of this paper. Prototype DAA algorithms have also been developed for alerting and maneuver guidance (referred to as guidance in this paper) research [9-11]. These developments enabled the RTCA Special Committee 228 (SC-228) to publish the Minimum Operational Performance Standards (MOPS) for DAA systems [12] and air-to-air radar [13] in 2017. The corresponding Technical Standard Orders (TSO), TSO-C211 and TSO-C212, were published by the Federal Aviation Administration (FAA) in October 2017. These standards, referred to as the Phase 1 MOPS, target UAS operations transitioning into, out of, or through class D, E (up to 18,000 ft MSL), and G airspace to or from class A airspace. A DAA system, according to the Phase 1 MOPS, contains surveillance components of Automatic Dependent Surveillance-Broadcast (ADS-B) In, airborne active surveillance that can interrogate transponders of nearby aircraft, and air-to-air radar that can detect aircraft with or without transponders. Traffic Alert and Collision Avoidance System (TCAS) II [14] is an optional component. Phase 2 work for extending the MOPS to additional UAS categories and operations is underway.

One of the Phase 2 objectives seeks an alternative DWC for UAS with non-cooperative aircraft, i.e., visual flight rules (VFR) aircraft without a broadcasting transponder. The DWC in the Phase 1 work was selected with considerations of interoperability with TCAS-II. To avoid triggering TCAS's resolution advisories during an encounter which leads to DAA maneuvers, the DWC was defined to encompass a vast majority of the TCAS alerting volume [1]. The resulting DWC is deemed very safe but may be unnecessarily large for encounters of UAS with noncooperative aircraft, which TCAS-II cannot detect and therefore need not be considered. Additionally, the airborne radar required under Phase 1 is heavy and consumes considerable power, which makes it difficult for smaller UAS to operate in compliance with the Phase 1 MOPS. A smaller DWC for non-cooperative aircraft would allow these UAS to equip with a lower-power, lighter weight airborne radar, provided the UAS restricted their operational speeds to values between 40 and 100 kts true airspeed (TAS). Four candidate DWCs were proposed for additional analysis in terms of their alerting performance and safety metrics [15].

Another Phase 2 objective considers extending the maximum UAS true airspeed defined in RTCA Document 365 (DO-365) from the current limit of $200 \mathrm{kts}$ to $291 \mathrm{kts}$ for Phase 1 compliant UAS. A true airspeed of $291 \mathrm{kts}$ is roughly equivalent to $250 \mathrm{kts}$ indicated airspeed at around 10,000 ft MSL. This 250-knot IAS value is notable because it is the FAA-imposed upper bound for aircraft flying under 10,000 ft MSL. If the Phase 1 MOPS is extended to $291 \mathrm{kts}$ for these large UAS, updated supporting information must be provided that considers higher closure rates between UAS and non-cooperative VFR traffic.

The Phase 1 airborne radar, documented in RTCA DO-366 [13], serves as the sole surveillance component for detecting non-cooperative intruders. This large, high-power radar shall detect a large non-cooperative intruder such as a King Air at a distance of $6.7 \mathrm{nmi}$. Both objectives described above have the effect of changing the required surveillance volume of the Phase 1 radar. If the required alerting time can be assumed to be independent of a DWC, a reduced DWC can potentially reduce the required surveillance volume. On the other hand, increasing the maximum UAS speed would have the effect of increasing the required surveillance volume so as to ensure sufficient alerting times for encounters with higher closure rates.

As a direct support of SC-228 Phase 2 MOPS work, this paper analyzes the combination of increased maximum UAS speed (291 kts TAS) and a reduced DWC size on the trade space for the alerting timeline and the radar's surveillance volume. DWC serves as an independent variable, with each of the four candidate values and the Phase 1 DWC tested individually. Results will inform the SC-228 of recommendations to surveillance requirements.

This paper is organized as follows: Section II provides additional background information of DWC, alerting, and guidance. Section III describes the experiment plan. Results are given in Section IV and additional discussion given in Section V. Section VI concludes this work.

\section{Background}

\section{A. Anatomy of a DWC Definition}

The DWC definitions discussed in this paper are defined by a group of four parameters. These parameters are: horizontal miss distance (HMD), modified tau ( $\left.\tau_{\text {mod }}\right)$ and its associated distance modifier (DMOD), and current altitude separation $(h)$. The horizontal miss distance is the horizontal distance between the UAS and another aircraft at the 
projected horizontal closest-point-of-approach (CPA). This projection is a state-based "dead-reckoning" trajectory that assumes both the UAS and the other aircraft will continue in their current direction of travel from their current relative position with their current relative vertical and horizontal velocities. The altitude separation is calculated at the aircrafts current time. When two aircraft are diverging, $\tau_{\bmod }$ is set to infinity. For two aircraft that are converging, $\tau_{\bmod }$ and the associated DMOD are shown in the equation below. Note that the range (r) used is the horizontal range without a vertical component, and the rate-of-change of the range $(\dot{r})$ is negative when aircraft are converging.

$$
\tau_{\text {mod }}= \begin{cases}\frac{-\left(r^{2}-D M O D^{2}\right)}{r \dot{r}}, & r>D M O D \\ 0, & r \leq D M O D\end{cases}
$$

The value of DMOD for each DWC definition in this paper is set to the value of the HMD threshold to avoid undesirable oscillations of alerts arising from the concavity of the DWC volume [20]. Additionally, when $r$ is less than DMOD, the value of $\tau_{\text {mod }}$ is set to zero. This ensures that when the UAS detects another aircraft violating the HMD criteria, the $\tau_{\text {mod }}$ criteria will also be violated.

\section{B. Alternative Detect-and-Avoid Well Clear Definition}

The original Phase 1 DWC was created as a single DWC definition for all encounters between a UAS and another aircraft. As discussed in the Introduction, that decision led to the size of the Phase 1 DWC as it needed to safely interact with TCAS II, which could be equipped on many cooperative aircraft. TCAS-II is a system that is designed to be a "last resort." As such, when a TCAS-II Resolution Advisory (RA) appears to a pilot, the pilot is expected to follow that advisory, no matter what other guidance the pilot might be receiving from other sources. The decision to make DAA alerts occur before potential TCAS-II RA's was to avoid potentially confusing situations in which a UAS pilot received a TCAS-II RA calling for a climb followed by a DAA alert asking for a descent. This requirement for TCAS compatibility pushed the radar range out to $6.7 \mathrm{nmi}$, as discussed in the Introduction, and made it too heavy and/or large for some of the smaller UAS.

The four alternative DWC definitions analyzed in this paper were candidate DWCs for encounters between noncooperative VFR traffic and UAS. These candidates were proposed based on analysis [15] of encounters representing operations of UAS that are smaller and cannot carry a Phase 1 compliant radar. The primary metrics considered were unmitigated collision risk and maneuver initiation range.

\section{DWC Candidate Definitions}

The DWC definitions discussed in this paper fall into two categories. The first is the DWC definition that was accepted by the FAA at the conclusion of "Phase 1" work by SC-228. This is referred to in the paper as the "Phase 1 DWC." The second category consists of four candidate DWC volumes that were being tested for implementation in "Phase 2," and are discussed in detail in Ref. 16. The threshold values of the DWC definition parameters for each of the five DWC's are given in the table below. In order for a loss of DAA well clear (LoDWC) to be declared, all of the parameter thresholds must be violated at the same time. Note that for DWC2, which has a $\tau_{\text {mod }}$ threshold of zero, LoDWC will only occur when the UAS and another aircraft are physically within the HMD and $h$ thresholds.

Table 1. DAA well-clear definitions and threshold parameter values

\begin{tabular}{cccc}
\hline DAA Well Clear Label & $\begin{array}{c}\text { HMD and DMOD } \\
\text { threshold (ft) }\end{array}$ & $\boldsymbol{\tau}_{\text {mod }}$ threshold (s) & $\boldsymbol{h}$ threshold (ft) \\
\hline Phase 1 DWC & 4000 & 35 & 450 \\
DWC 1 & 2000 & 15 & 450 \\
DWC 2 & 2200 & 0 & 450 \\
DWC 3 & 1500 & 15 & 450 \\
DWC 4 & 2500 & 25 & 450
\end{tabular}

The candidate DWC definitions use smaller parameters than the Phase 1 DWC for HMD and $\tau_{\text {mod, }}$ but keep the same threshold value of $h$. This is in large part because the current airspace is structured such that 500 feet is the minimum vertical separation that aircraft are generally allowed to have below 10,000 feet MSL. So if the threshold value of $h$ was increased to 500 feet, there would be a risk of legitimate traffic that was already adequately separated, according to convention, triggering a DAA alert and pilot response. Due to some preliminary, unpublished study 
results that showed limited benefit to alerting metrics, an expected adverse effect on safety metrics, and discussions with subject matter experts from SC-228, the threshold value of $h$ value is not decreased in the candidate definitions, either.

\section{Motivation}

As discussed previously, part of the motivation for this work came from discussions about whether the Phase 1 DWC definition was necessary for non-cooperative VFR encounters, as that DWC was sized primarily by cooperative VFR constraints. Additionally, using one of the candidate DWC definitions from Phase 2 would enable the use of a single DWC definition for non-cooperative VFR encounters for all UAS. It should be noted that this investigation is only for the non-cooperative DWC definition. The DWC definition for cooperative VFR encounters is already the same for both Phase 1 and Phase 2 UAS, due to the relatively low cost, size, weight, and power requirements of cooperative sensors. It should also be noted that, even if one of the candidate DWC definitions was adopted for noncooperative VFR encounters by Phase 1 UAS, those UAS would still be expected to carry a Phase 1 compliant radar.

As stated in the Introduction, using one of the smaller candidate DWC definitions could potentially decrease the required surveillance volume of the Phase 1 radar, increase the maximum allowable Phase 1 UAS airspeed at altitudes below 10,000 ft MSL, or some combination of both. However, prioritizing the decrease in radar range or the increase in allowable UAS speed is beyond the scope of this paper, and would be determined by SC-228.

In order to apply one of the candidate DWC definitions from Phase 2 to Phase 1 compliant UAS, it must be shown that the reduced DWC will not adversely affect the safety and alerting metrics. The effect of the reduced DWC on safety metrics is covered in another paper [17]. This paper uses simulation results that do not include any pilot actions to maintain DWC, referred to as "unmitigated" simulations, to determine the effects of a smaller DWC on alerting metrics for the Phase 1 UAS operations.

This paper describes part of an effort to identify the effects of modifying the DWC for Phase 1 compliant UAS in an encounter with non-cooperative VFR traffic. The specific focus of this paper is on the alerting metrics.

\section{Experiment Plan}

The experiment that underpins this paper leveraged the simulation architecture that was used in a previous study [16]. The data come from a fast-time simulation that used a full day of NAS-wide real VFR traffic data and projected UAS missions and trajectories. The UAS missions and trajectories are filtered to capture only the trajectories that are between 500 feet AGL and 10,999 feet MSL.

\section{A. Encounter Generation}

The VFR traffic was extracted from ground-based radar tracks recorded on 21 non-contiguous days in 2012 by the 84th RADAR Evaluation Squadron. The tracks contain non-cooperative tracks and cooperative tracks from vehicles "squaking" a 1200 transponder code (standard transponder setting for cooperative VFR aircraft). These were processed to remove measurement noise and produce continuous trajectories, which were fed into the simulation. It is estimated that roughly $15 \%$ of VFR traffic is non-cooperative [12], but due to the low number of non-cooperative VFR tracks that could be extracted, some of the lower speed cooperative VFR tracks were used as surrogates for noncooperative traffic in this study. This substitution was made in the previous study [16], and is considered reasonable due to the similarity in the airspeed, turn rate, and accelerations of both cooperative and non-cooperative VFR aircraft when true airspeeds are below 170 knots [18]. The speed distributions of the UAS and VFR traffic are shown below in Fig. 1a and Fig .1b, respectively, while the altitude distributions of the UAS and VFR traffic are shown in Fig. 1c and Fig. 1d. 

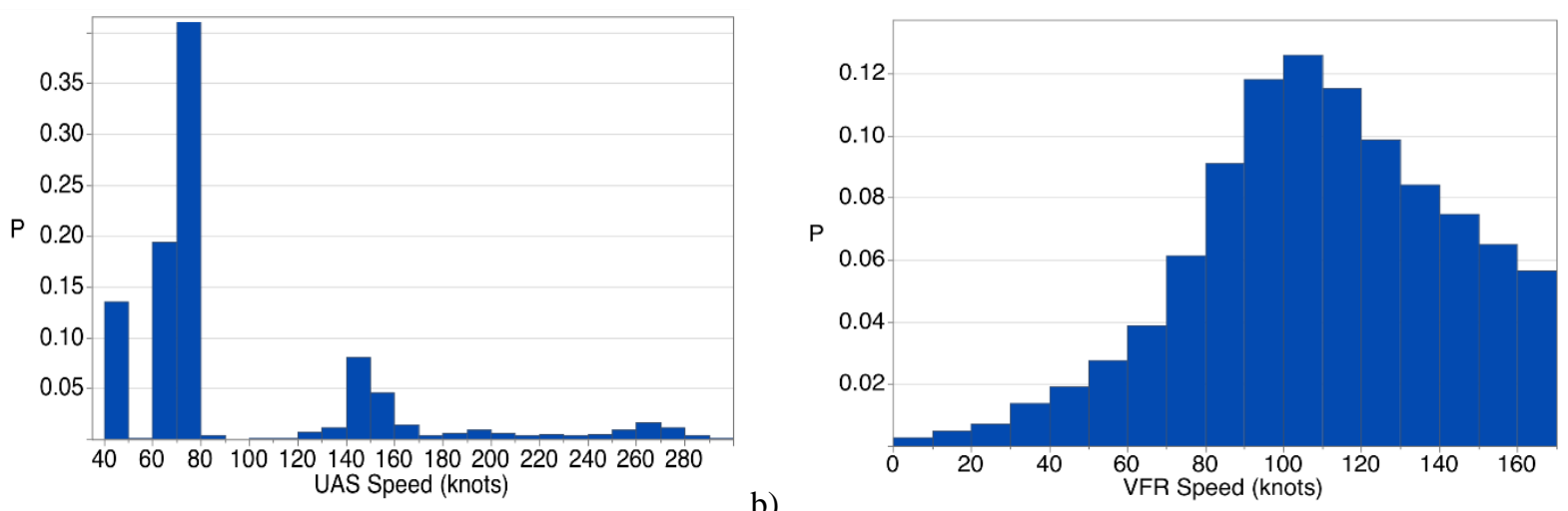

a)

b)
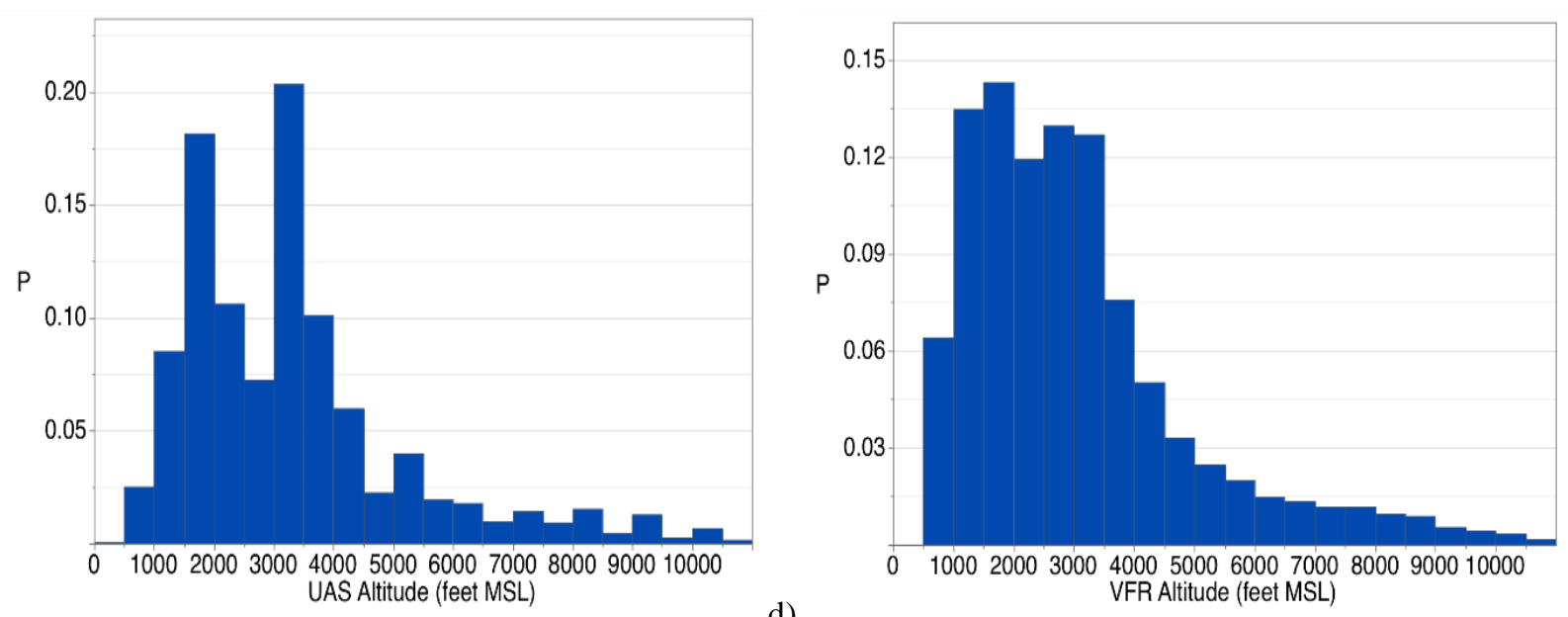

c)

d)

Fig 1. Histograms with probability of true airspeeds for a) UAS and b) non-cooperative VFR flights, and probability of altitude for c) UAS and d) non-cooperative VFR flights in the simulation.

The UAS traffic is from a NAS-wide set of trajectories created from demand estimations and potential mission profiles built from opinions of subject matter experts [19]. All UAS speeds were considered, but the UAS altitude was restricted to values between $500 \mathrm{ft}$ AGL and 10,999 ft MSL. 10,000 ft MSL is the upper bound at which a UAS could encounter non-cooperative VFR traffic but is raised to $10,999 \mathrm{ft}$ MSL to include a few UAS missions flying slightly above 10,000 ft MSL.

These two sets of trajectories were run through a NAS-wide, fast-time simulation called the Java Architecture for DAA Extensibility and Modeling (JADEM) [9] to extract encounters that feature one UAS and one non-cooperative VFR flight that are close enough to potentially trigger the DAA alerting logic. Each encounter has only one pair of aircraft, and thus are referred to as "pairwise encounters" for the rest of this paper. In total, there were 94,093 of these pairwise encounters extracted for this study. Of those encounters, 505 led to a near mid-air collision, which is defined by two aircraft passing within $500 \mathrm{ft}$ horizontally and $100 \mathrm{ft}$ vertically of each other. Those 94,093 pairwise encounters were then run, unmitigated, through each point of the simulation test matrix to determine the effects of different DWC definitions, air-to-air radar ranges, and radar horizontal and vertical fields of regard on a group of alerting metrics. The alerts themselves were generated by the open-source Detect-and-AvoID Alerting Logic for Unmanned Systems (DAIDALUS) [19].

DAIDALUS can produce alerts at three levels. In order of increasing severity, they are Preventive, Corrective, and Warning alerts. For Preventive alerts, no action is required by the UAS pilot. For a Corrective alert, the pilot is expected to maneuver, but needs to coordinate with an air-traffic controller before maneuvering to avoid the other aircraft. For a Warning alert, the pilot is expected to maneuver to avoid the other aircraft as soon as possible, without a coordination requirement. DIADALUS can also provide guidance for the pilot during these alerts, but these studies were unmitigated and so no maneuver was executed upon guidance. The conflict zone the alerting and guidance protects is based on each DWC with its HMD threshold buffered by a factor of 1.519. The buffer gives the system a few seconds to alert against aircraft suddenly maneuvering towards the UAS. Corrective and warning alerts are issued 
if an intruder is predicted, with a constant velocity assumption, to enter the conflict zone within 60 and 30 seconds, respectively.

\section{B. Variables and Metrics}

The independent variables in this study are DWC type, radar range, radar bearing limits, and radar azimuth limits. The results are further broken down by UAS speed range, to highlight specific metrics that are strongly affected by UAS speed. As a final filter, only the encounters that led to an unmitigated loss of DWC are included in the metrics. This is to ensure that each alert that is processed is one that would have required a pilot's input to avoid. Note that the Phase 1 MOPS requires a radar with 15 degrees elevation (vertical field of regard) and 110 degrees bearing (horizontal field of regard). The test matrix is shown below in Table 2. In all, there were 60 test cases, with each test using a single test value from each of the four test parameters.

Table 2. Test matrix

\begin{tabular}{lll}
\hline Test Parameter & Units & Tested Values \\
\hline DWC Type & n/a & Phase 1 DWC, DWC1, \\
& & DWC2, DWC3, DWC4 \\
Radar range & nautical miles & $4,6,8$ \\
Horizontal field of regard & degrees from centerline & $+/-110,+/-180$ \\
Vertical field of regard & degrees from centerline & $+/-15,+/-90$
\end{tabular}

The dependent variables that were examined included the average alert time for Corrective and Warning alerts (time between the first alert and a LoDWC), the range from the UAS at which an alert first occurred, and the relative heading and elevation angles of the two aircraft at the time of first alert. Only the Corrective alerts are included in this paper, as they occur farther away from the UAS and drive the air-to-air radar requirements. The metrics for Warning alerts showed similar trends to the metrics for Corrective alerts. Preventive Alerts, which are included in the MOPS for Phase 1 UAS, are not included in this study, as they do not require pilot action.

Sensor uncertainty, DAA guidance, and pilot action times are not considered in this paper, but will be examined in future studies.

\section{Data and Results}

As stated in the previous section, the results presented in this study focus primarily on the Corrective alerts and the effect of the independent variables on the Corrective alert timeline. This is because Corrective alerts, which still require pilot action, have significantly longer average alerting time requirements than Warning alerts, and are impacted by restricted radar range more heavily than warning alerts. The field-of-regard limitations had similar effects on both the Corrective and Warning alerts, so the Corrective alert data are shown for continuity. The only exception is Table 3 , below, which includes average Warning alert time. 
Table 3. Average alert times for corrective and warning alerts for UAS flying between 40 and 200 kts TAS and radar with full horizontal field of regard and full vertical field of regard

\begin{tabular}{c|c|c|c|c|c|}
\multicolumn{2}{c}{ Range (nmi) } & \multicolumn{1}{c}{$\begin{array}{c}\text { Corrective } \\
\text { DWC Label }\end{array}$} & $\begin{array}{c}\text { Standard Error of the } \\
\text { Alert Time (s) }\end{array}$ & $\begin{array}{c}\text { Warning } \\
\text { Alert Time (s) }\end{array}$ & $\begin{array}{c}\text { Standard Error of the } \\
\text { Mean (Warning) }\end{array}$ \\
\hline 4 & Phase1DWC & 48.04 & 0.18 & 34.01 & 0.130 \\
\hline 4 & DWC1 & 51.89 & 0.24 & 32.65 & 0.156 \\
4 & DWC2 & 57.54 & 0.21 & 35.27 & 0.132 \\
4 & DWC3 & 49.30 & 0.28 & 30.81 & 0.18 \\
\hline 4 & DWC4 & 49.36 & 0.22 & 32.39 & 0.15 \\
\hline 6 & Phase1DWC & 56.00 & 0.17 & 35.28 & 0.13 \\
\hline 6 & DWC1 & 54.05 & 0.24 & 32.65 & 0.16 \\
6 & DWC2 & 58.41 & 0.21 & 35.27 & 0.13 \\
6 & DWC3 & 51.15 & 0.28 & 30.80 & 0.18 \\
6 & DWC4 & 53.53 & 0.22 & 32.69 & 0.15 \\
\hline 8 & Phase1DWC & 57.00 & 0.17 & 35.28 & 0.13 \\
\hline 8 & DWC1 & 54.15 & 0.24 & 32.65 & 0.16 \\
8 & DWC2 & 58.41 & 0.21 & 35.27 & 0.13 \\
8 & DWC3 & 51.22 & 0.28 & 30.80 & 0.18 \\
8 & DWC4 & 53.89 & 0.22 & 32.69 & 0.15 \\
\hline
\end{tabular}

Table 3 shows the average alert times for both corrective and warning alerts for the four candidate definitions as well as the Phase 1 DWC. The standard error of the averages is also included. In this table, the air-to-air radar had full horizontal field of regard (+/- 180 degrees) and full vertical field of regard (+/- 90 degrees). The UAS true airspeed values were between 40 and 200 knots TAS. Examination of the corrective alerts show that limiting the radar range to $4 \mathrm{nmi}$ shortens the average corrective alert time for all DWCs, and that DWC2 had the longest average corrective time of all DWCs at all radar ranges. Overall, these results show that removing the $\tau_{\text {mod }}$ component completely from a DWC definition (DWC2) seems feasible. These results also show that only DWC2 and the Phase 1 DWC met the MOPS requirements for average corrective and warning alert times (55 seconds for corrective, 25 seconds for warning).

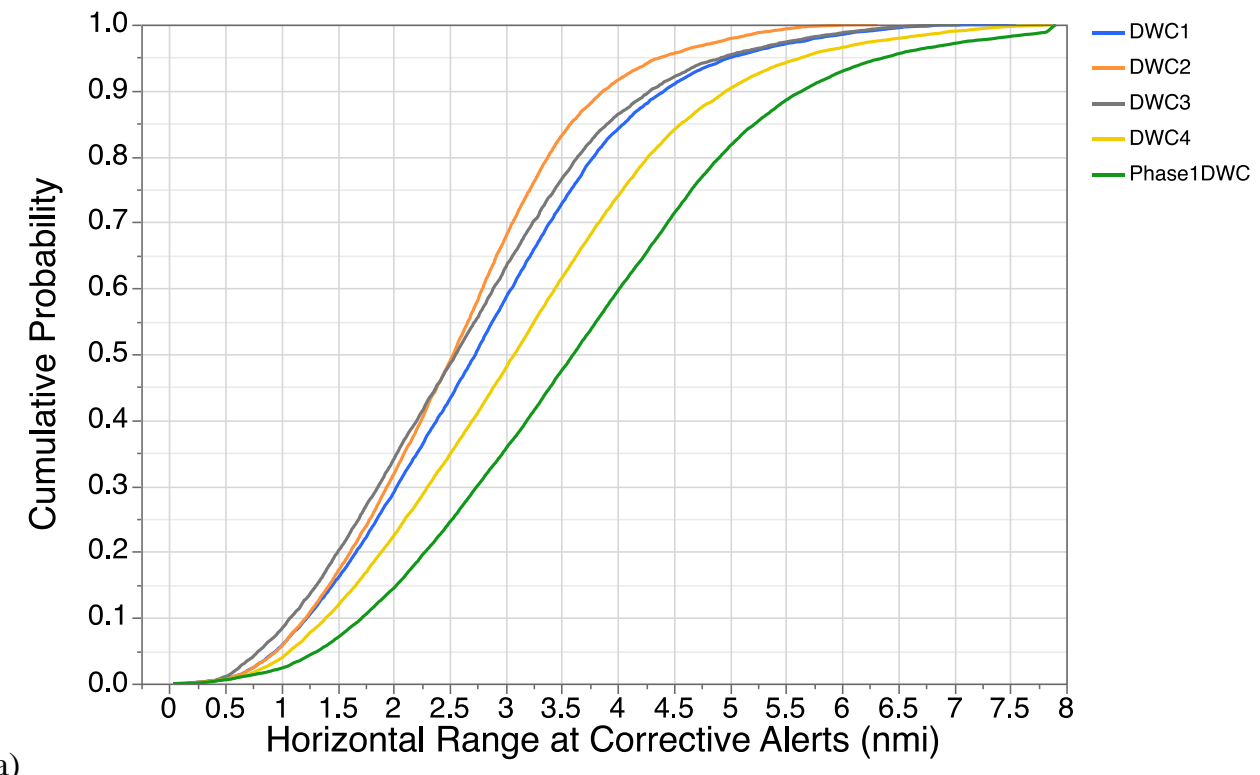




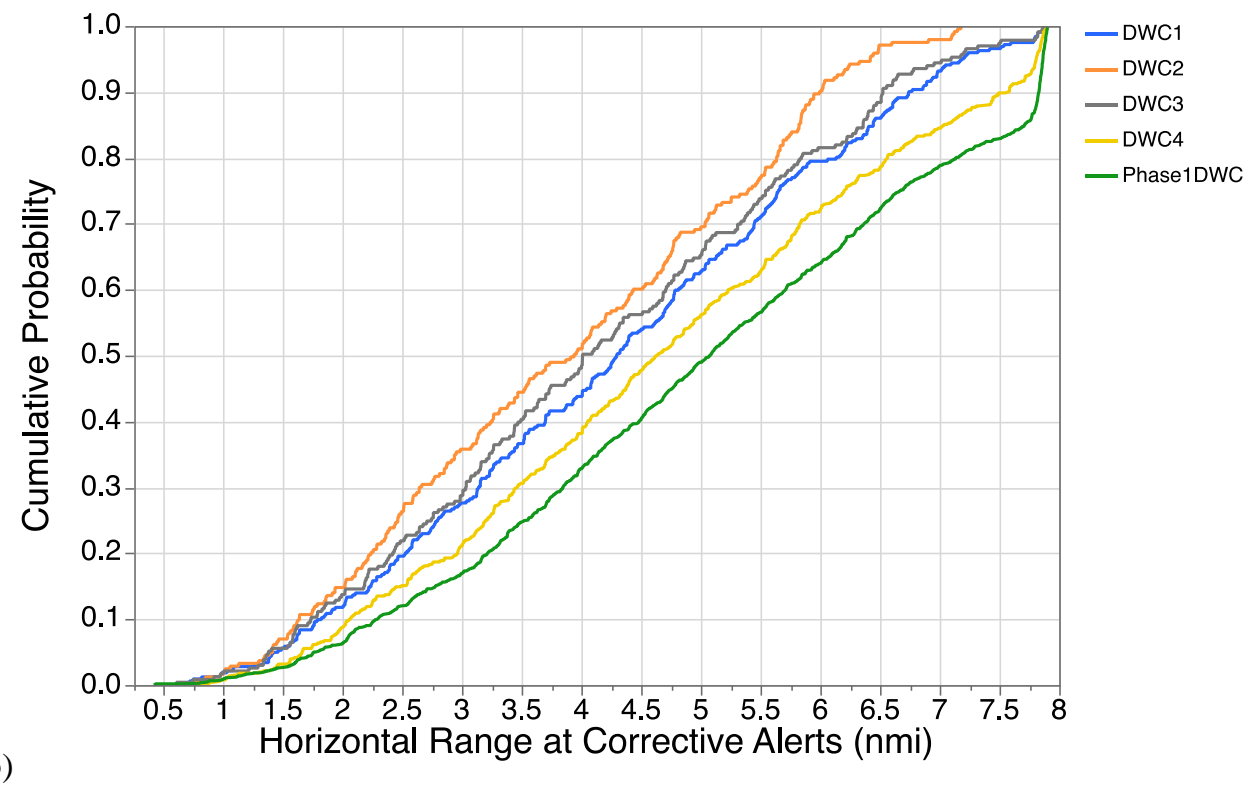

\section{Fig 2. Cumulative distribution of range at the point of first corrective alert for UAS flying with a true airspeed a) between 40 and 200 knots, and b) between 200 and 291 knots.}

Figure $2 \mathrm{a}$ and $2 \mathrm{~b}$ show the cumulative distribution of the range at the point of first corrective alert for each encounter. The vertical axis is the cumulative probability that a given encounter will have its maximum potential corrective alert time at a certain range. This does not mean that the encounter will have the desired 55 seconds of corrective alert time, as a sudden maneuver could limit the "maximum potential value" of a corrective alert to just a few seconds, depending on the encounter geometry and relative velocities of the UAS and VFR aircraft. These curves are generated from data that has a maximum radar range of $8 \mathrm{nmi}$, and has full horizontal and full vertical fields of regard. This allows the comparison of DWC performance based exclusively on what the range value was when the first corrective alert was recorded. The difference in the smoothness of the curves in Fig. 2a versus Fig. $2 b$ is due to a difference in the number of pairwise encounters in each data set, as each DWC's curve only includes encounters that lead to a LoDWC. For Fig. 2a, the number of pairwise encounters varies from 7,402 (DWC3) to 23,735 (Phase 1 DWC), while for Fig. 2b, the number of encounters is between 233 (DWC3) to 1074 (Phase 1 DWC). As an example of how to interpret this data, look at the Phase 1 DWC curve in Fig. 2a. The curve crosses a probability of 0.6 at 4 nmi, which means that $60 \%$ of the encounters that lead to a LoDWC will have their maximum potential corrective alert duration (actual value varies by encounter) if the radar range is $4 \mathrm{nmi}$, while the other $40 \%$ of the encounters will have some truncation of their alerting timeline. As these data are from an encounter set that includes maneuver VFR traffic and maneuver UAS, it does not show which encounters will have at least 55 seconds of corrective alert time. Instead, these figures, as well as Fig. 3 and Fig. 4 below, show what percentage of corrective alerts will have their timelines truncated, regardless of what the maximum value might be.

Figure 2 a shows data for the UAS that are flying between 40 and 200 knots TAS. These results show that all of the candidate DWC definitions have a lower radar range requirement than the Phase 1 DWC, with DWC2 requiring the least range. This is an expected result considering the reduced HMD and $\tau_{\text {mod }}$ requirements of the candidate DWC's. Examining the Phase 1 DWC curve in combination with the MOPS requirement of $6.7 \mathrm{nmi}$ for radar range shows that close to $97 \%$ of corrective alerts will alert at the maximum possible distance if the minimum radar range is used with the Phase 1 DWC. If DWC2 was implemented for these encounters, the range at which $97 \%$ of the corrective alerts would be expected to have their maximum potential timeline is $5 \mathrm{nmi}$. This implies the radar range requirement could be relaxed from $6.7 \mathrm{nmi}$ for Phase 1 UAS if DWC2 was adopted for non-cooperative VFR traffic without increasing the percentage of encounters that have less than the maximum possible corrective alert duration.

Figure $2 \mathrm{~b}$ shows the data for UAS that are flying between 200 and 291 knots TAS. These higher-speed UAS would be required to reduce their speed to 200 knots TAS under the current MOPS. Examining the Phase 1 DWC curve shows this is a logical restriction, as less than $80 \%$ of encounters have their maximum potential corrective alert duration with this DWC definition and the current radar minimum range of $6.7 \mathrm{nmi}$. The relative sparsity of the data compared to the curve in Fig. 2a is due to the very limited number of UAS that are flying above 200 kts TAS in this data set (see Fig. 1a). It is not possible to determine what the required radar range would need to be increased to so 
that $97 \%$ of corrective alerts have their maximum potential duration, as the tested radar range for this study only went as high as $8 \mathrm{nmi}$. However, by using one of the smaller candidate DWC definitions, it should be possible to maintain the original $97 \%$ maximum potential duration corrective alert rate without significantly increasing the radar range requirement. In fact, the current requirement of $6.7 \mathrm{nmi}$ combined with DWC2 would allow UAS flying between 200 and 291 knots TAS to maintain the same percentage of corrective alerts that are at maximum duration as UAS that are using the current Phase 1 DWC with true airspeeds restricted to below 200 knots.

Overall, these two figures imply that changing the DWC from the Phase 1 definition to one of the candidate DWCs, and their smaller alerting volume, could allow for reduced radar range requirement or increased allowable UAS speed or some combination of both without sacrificing alerting time.

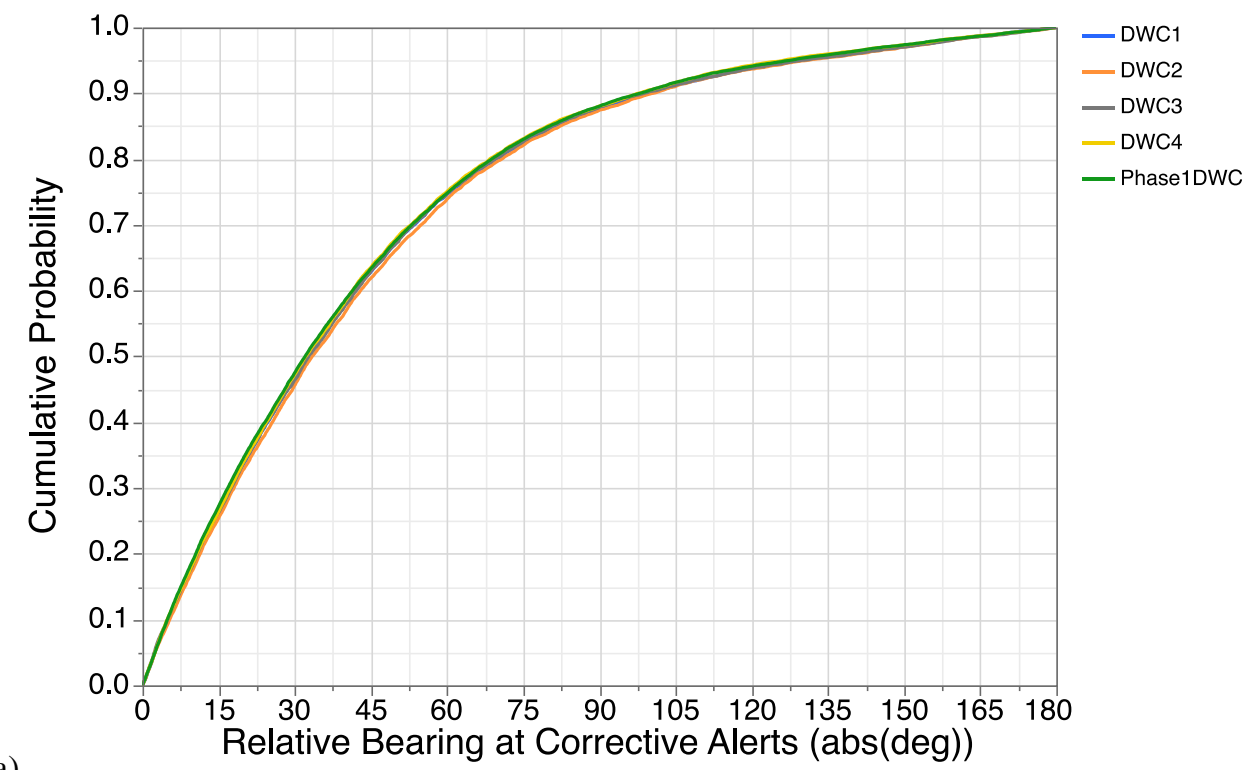

a)

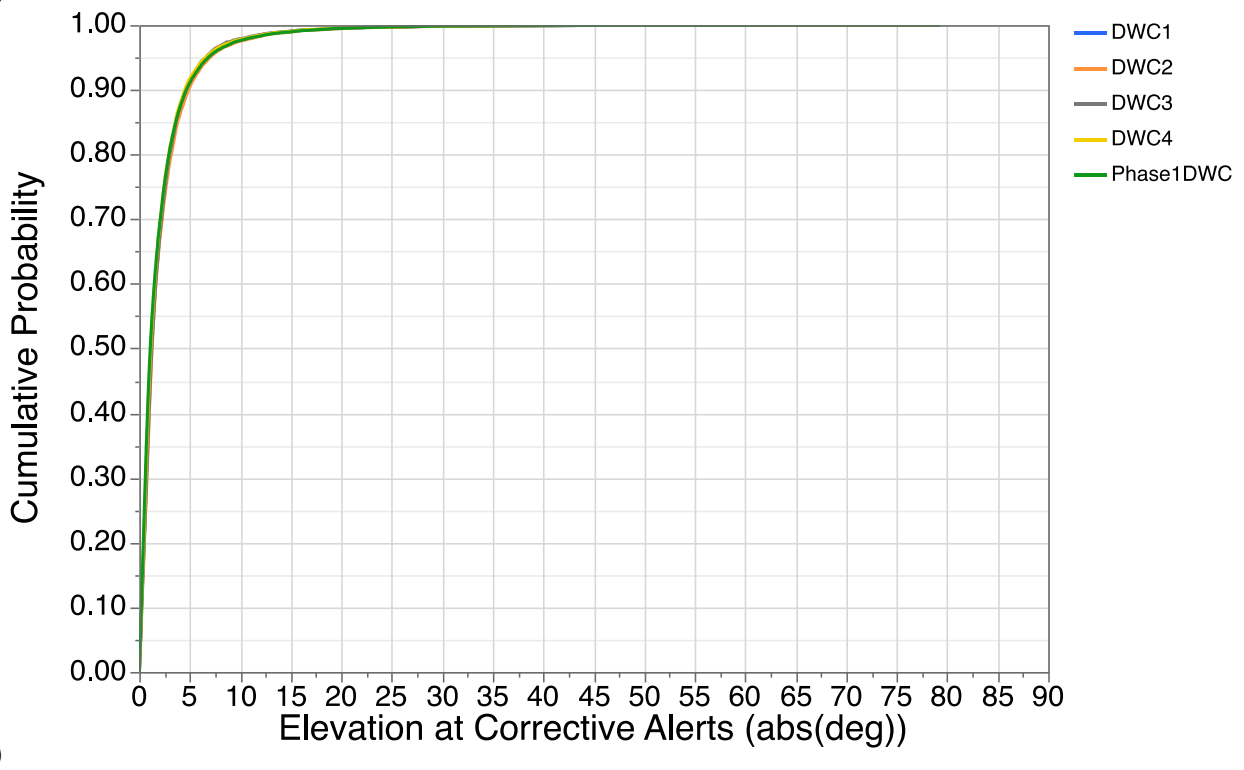

Fig 3. Cumulative distribution of a) relative bearing and b) relative elevation at the point of first corrective alert for a UAS with true airspeed between 40 and 200 knots.

Figure 3 shows that both the bearing and elevation range curves are relatively independent of the DWC definition. These curves show the percentage of LoDWC encounters that achieve their maximum potential corrective alert timeline if the radar is limited to the specified bearing or elevation range. The number of pairwise encounters in these data varies between 7,402 and 23,735, depending on DWC type. As discussed in Table 2, the angular ranges specified 
on the $\mathrm{x}$-axis of Fig. 3a and Fig. $3 \mathrm{~b}$ are the symmetric ranges either to the left and right, or above and below the centerline of the UAS. As an example, a bearing range of 90 on Fig. 3a means the air-to-air radar can track targets up to $+/-90$ degrees horizontally from the direction of travel. The requirements in the current MOPS call for $+/-110$ degrees of horizontal and $+/-15$ degrees of vertical field of regard. These results show that the percentage of corrective alerts that had their timeline truncated due to horizontal and vertical field-of-regard constraints was not significantly affected when one of the new candidate DWCs was used instead of the Phase 1 DWC. Note that Fig. 3a shows that +/-110 degrees of horizontal field of regard allowed maximum possible corrective alert duration in $93 \%$ encounters.

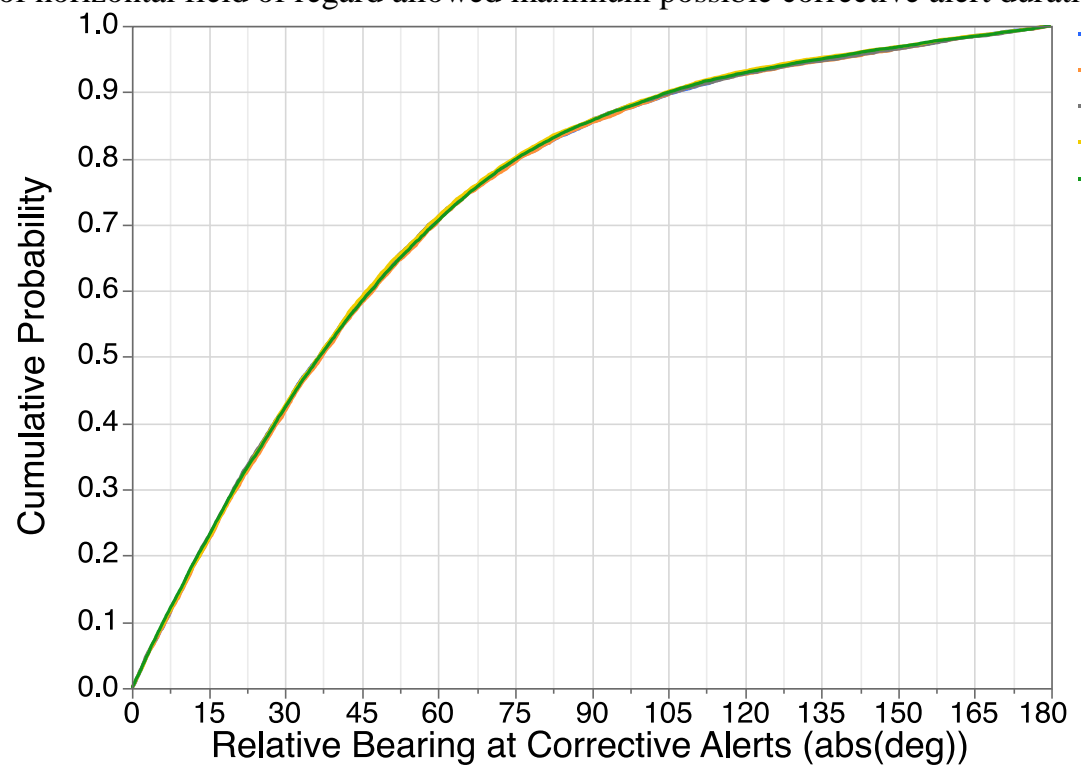

a)

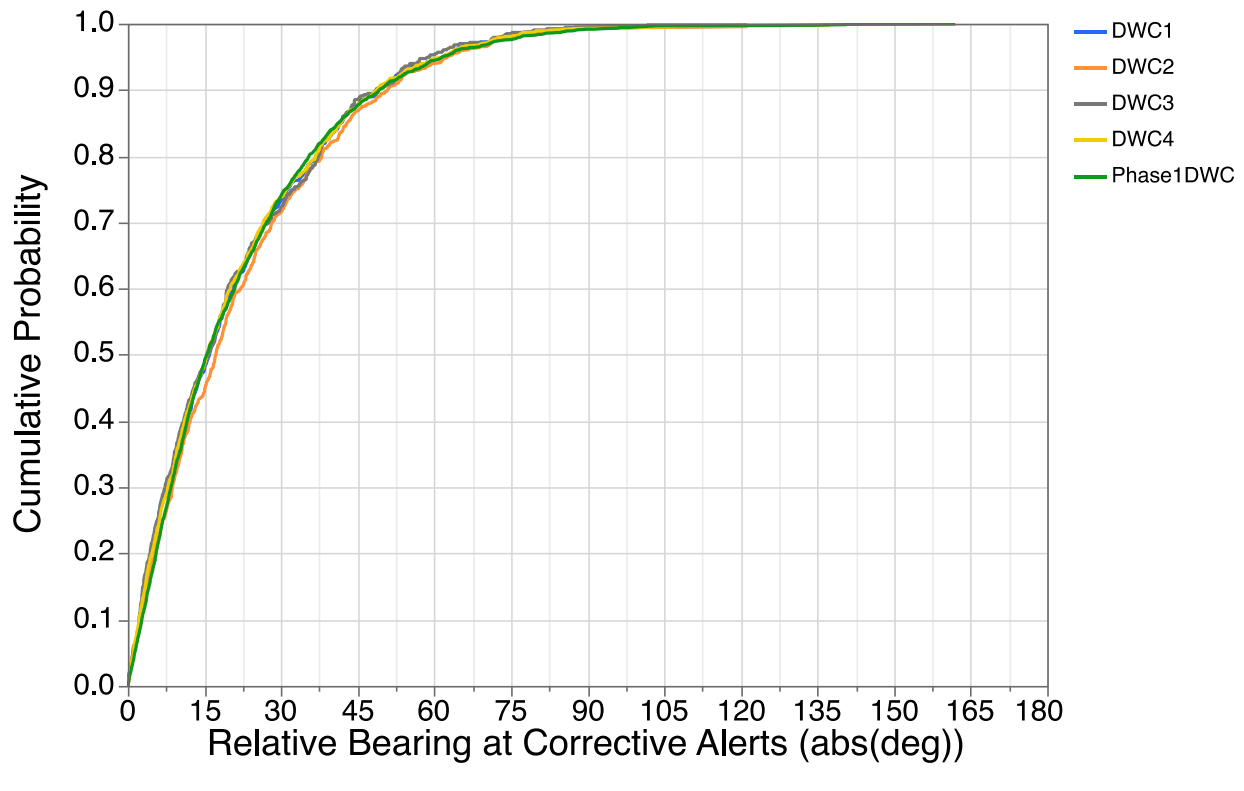

Fig. 4 Relative bearing at the position of first corrective alert for UAS with a radar range of $8 \mathrm{nmi}$ and a true airspeed a) between 40 and 100 knots, and b) between 100 and 150 knots

Figure 4 further parses the data presented in Fig. 3a. Figure 4 shows the strong effect that UAS speed had on the realtive bearing at which the first alert occured. Fig. 4a shows the relative bearing at first corrective alert when the UAS had a true airspeed between 40 and 100 knots, while Fig. $4 \mathrm{~b}$ shows the same plot but with the true airspeed between 100 and 150 knots. As in previous figures, the difference in the smoothness of the curves is due to the difference in the number of pairwise encounters in each speed bin. The number of pairwise encounters for Fig. 4a is between 6,292 and 19,549, while for Fig. 4b it is between 532 and 2,175, depending on the DWC being tested. The major difference in the slopes of the two figures is because in Fig. 4a, the UAS was generally slower than the VFR 
traffic it encounted, while in Fig. 4b the UAS was generally faster. This is because the non-cooeprative VFR traffic airspeed peaked around 100 knots, as was shown in Fig. 1b. When a UAS is flying slower than the non-cooperative VFR traffic, it is much more likely that the VFR aircraft will aproach the UAS from the side or slightly behind. This difference in encounter gemoetry is why the field-of-regard of the radar, rather than its range, seems to have the largest impact on the alerting timeline for UAS flying below 100 knots TAS. This same effect was noticed when the low CSWaP UAS were studied, as they were a subset of the UAS in Fig. 4a. The data for UAS true airspeeds above 150 knots are very similar to Fig. 4b. Overall, Fig. 4 shows that, in order to allow maximum potential corrective alert duration in $93 \%$ of encounters, a horizontal field of regard of +/- 125 degrees is required for UAS below $100 \mathrm{kts}$ TAS, and around +/- 50 degrees is required for UAS at and above $100 \mathrm{kts}$ TAS.

\section{Discussion and Future Work}

Previous work has led to a recommendation that the Low C-SWAP radar have a horizontal field of regard of $+/-$ 140 degrees from centerline[16], instead of the Phase 1 value of $+/-110$, with the goal of allowing $95 \%$ of encounters to have their maximum possible alert duration. While the aggregate data presented in Fig. 2 a suggest that $+/-110$ degrees is sufficient to allow $93 \%$ of encounters to have their maximum possible corrective alert duration, the data in Fig. 3a implies that the low-speed Phase 1 UAS, which make up a majority of the UAS flying below 10,000 feet, would require closer to +/-125 degrees to allow $93 \%$ and $+/-135$ degrees to allow $95 \%$ of encounters to have their maximum possible corrective alert duration. These Phase 1 UAS flying below 100 kts TAS are the primary drivers of the horizontal field-of-regard requirement for the agreggated data set of Phase 1 UAS flying between 40 and $200 \mathrm{kts}$ TAS. For these low-speed UAS, the horizontal field of regard, rather than the range of the radar, appears to have the largest impact on the alerting timeline. To that end, should SC-228 decide to pursue allowing shorter radar ranges for Phase 1 UAS, it will likely be beneficial to also investigate increasing the radar horizontal field of regard requirement, as it currently only allows $91 \%$ of the corrective alerts for Phase 1 UAS flying below 100 kts TAS to have their maximum possible duration.

The work presented in this paper is aimed at understanding the effects of changing the DWC definition for noncooperative aircraft encountered by Phase 1 UAS on alerting metrics. To fully understand the overall effects of changing the DWC definition, a few more pieces are needed, including the effect on safety metrics, the impact of sensor uncertainty, and how the new DWC would affect pilots and air traffic controllers.

A study examining the impact of sensor noise and error on the alerting and guidance when using a smaller DWC is currently in software development stages. There are also plans to explore pilot-related questions such as pilot response times and pilot acceptability in future human-in-the-loop studies.

\section{Conclusion}

This paper examined four candidate DWC definitions as potential replacements for the DWC definition currently in MOPS for use with non-cooperative VFR aircraft. These candidate definitions were taken from "Phase 2" studies, which were focused on small UAS that could not carry the radar required in the Phase 1 MOPS.

The alerting metrics studied in this paper suggest that using the new, candidate DWC definitions from Phase 2 would not adversely affect the alerting metrics of a Phase 1 UAS when encountering non-cooperative VFR traffic. Further, by using DWC2 (HMD of 2200 feet, h of 450 feet, and $\tau_{\text {mod }}$ of 0 ), the radar requirement from Phase 1 could potentially be reduced to $5 \mathrm{nmi}$ while keeping the same percentage of maximum duration corrective alerts as the current, Phase 1 DWC. Alternatively, if the radar requirement is kept at $6.7 \mathrm{nmi}$, it may be possible to increase the allowable UAS speed range from 200 knots TAS to 291 knots TAS without adversely affecting the alerting timeline. This would more closely align with the FAA's limit of 250 knots indicated airspeed at 10,000 feet MSL. Whether allowing increased UAS speeds below 10,000 feet or reducing radar range requirements has higher priority is a decision for SC-228.

DWC2 either matched or exceeded the alerting metric requirements from Phase 1, and had the lowest radar range requirement of all candidate DWC definitions. That definition is recommended by the authors for any modification of non-cooperative DWC in the Phase 1 MOPS. Additionally, DWC2 was selected by RTCA SC-228 in March 2019 as the DWC for encounters between Low C-SWaP UAS and non-cooperative VFR aircraft. Therefore, selecting DWC2 for Phase 1 UAS would also create a single DWC definition for UAS, regardless of size, in an encounter with a noncooperative VFR aircraft between $500 \mathrm{ft}$ AGL and 10,000 ft MSL. 


\section{References}

[1] Cook, S. P., Brooks, D., Cole, R., Hackenberg, D., and Raska, V., "Defining Well Clear for Unmanned Aircraft Systems," Proceedings of AIAA Infotech@ Aerospace, AIAA, 2015.

[2] Johnson, M., Mueller, E. R., and Santiago, C., "Characteristics of a Well Clear Definition and Alerting Criteria for Encounters between UAS and Manned Aircraft in Class E Airspace," Eleventh UAS/Europe Air Traffic Management Research and Development Seminar, 2015, pp. 23-26.

[3] Walker, D., "FAA Position on Building Consensus Around the SARP Well-Clear Definition," RTCA Special Committee 228, 2014.

[4] Murphy, J. R., Hayes, P. S., Kim, S. K., Bridges, W., and Marston, M., "Flight Test Overview for UAS Integration in the NAS Project," AIAA Atmospheric Flight Mechanics Conference, AIAA SciTech, 2016.

[5] Lee, S. M., Park, C., Thipphavong, D. P., Isaacson, D. R., and Santiago, C., "Evaluating Alerting and Guidance Performance of a UAS Detect-And-Avoid System," NASA Ames Research Center, 2016.

[6] Smearcheck, S., Calhoun, S., Adams, W., Kresge, J., and Kunzi, F., "Analysis of Alerting Performance for Detect and Avoid of Unmanned Aircraft Systems," IEEE/ION Position, Location and Navigation Symposium (PLANS), 2016, pp. 710-730.

[7] Rorie, R. C., Fern, L., and Shively, J., "The Impact of Suggestive Maneuver Guidance on UAS Pilot Performing the Detect and Avoid Function," AIAA InfoTech@ Aerospace Conference, AIAA SciTech, 2016.

[8] Fern, L., Rorie, R. C., Pack, J. S., Shively, R. J., and Draper, M. H., "An Evaluation of Detect and Avoid (DAA) Displays for Unmanned Aircraft Systems: The Effect of Information Level and Display Location on Pilot Performance," Proceedings of 15th AIAA Aviation Technology, Integration, and Operations Conference, 2015.

[9] Abramson, M., Refai, M., and Santiago, C., "A Generic Resolution Advisor and Conflict Evaluator (GRACE) in Applications to Detect-And-Avoid (DAA) Systems of Unmanned Aircraft," Proceedings of the 17th AIAA Aviation Technology, Integration, and Operations (ATIO) Conference, 2017.

[10] Muñoz, C., Narkawicz, A., Hagen, G., Upchurch, J., Dutle, A., Consiglio, M., and Chamberlain, J., "DAIDALUS: Detect and Avoid Alerting Logic for Unmanned Systems,” 34th Digital Avionics Systems Conference (DASC), IEEE/AIAA, 2015, pp. 5A1-1.

[11] Suarez, B., Kirk, K., and Theunissen, E., "Development, Integration and Testing of a Stand-Alone CDTI with Conflict Probing Support,” Infotech@ Aerospace 2012, 2012, p. 2487. doi:10.2514/6.2012-2487, URL https://arc.aiaa.org/doi/abs/10.2514/6.2012-2487.

[12] Minimum Operational Performance Standards (MOPS) for Detect and Avoid (DAA) Systems, DO-365, RTCA. Inc., 2017.

[13] Minimum Operational Performance Standards (MOPS) for Air-to-Air Radar for Traffic Surveillance, DO-366, RTCA. Inc., 2017.

[14] "Introduction to TCAS II Version 7.1," Federal Aviation Administration (FAA), 2011. URL http://www.faa.gov/documentLibrary/media/Advisory_Circular/TCAS\%20II\%20V7.1\%20Intro\%20booklet.pdf.

[15] Wu, M. G., Cone, A. C., Lee, S., Chen, C., Edwards, E. W. M., and Jack, D. P., "Well Clear Trade Study for Unmanned Aircraft System Detect and Avoid with Non-Cooperative Aircraft," 18th AIAA Aviation Technology, Integration, and Operations Conference, 2018.

[16] Wu, M. G., Cone, A. C., Lee, S., "Detect and Avoid Alerting Performance with Limited Surveillance Volume for NonCooperative Aircraft," AIAA SciTech Forum, 2019.

[17] Chen, C. C., Gill, B., Edwards, M. W. M., Smearcheck, S., Adami, T., Calhoun, S., Wu, M. G., Cone, A. C., Lee, S., "Defining Well Clear Separation for Unmanned Aircraft Systems Operating with Noncooperative Aircraft," 19 ${ }^{\text {th }}$ AIAA Aviation, Technology, Integration, and Operations Conference, 2019.

[18] Weinert, A. J., Harkleroad, E. P., Griffith, J., Edwards, M. W., and Kochenderfer, M. J., "Uncorrelated Encounter Model of the National Airspace System, Version 2.0," Tech. rep., MIT Lincoln Laboratory, Lexington, Massachusetts, Aug. 2013. URL www.dtic.mil/cgi-bin/GetTRDoc?Location=U2\&doc=GetTRDoc.pdf\&AD=ADA589697.

[19] Ayyalasomayajula, S., Sharma, R., Wieland, F., Trani, A., Hinze, N., and Spencer, S., "UAS Demand Generation Using Subject Matter Expert Interviews and Socio-Economic Analysis," Proceedings of the AIAA Aviation Conference, 2015.

[20] Muñoz, C, and Narkawicz, A., "Formal Analysis of Extended Well-Clear Boundaries for Unmanned Aircraft", NASA Formal Methods Symposium pp. 221-226, 2016. 